\title{
Vasopressin/Oxytocin-Related Signaling Regulates Gustatory Associative Learning in C. elegans
}

\author{
Authors: Isabel Beets ${ }^{1}$, Tom Janssen ${ }^{1}$, Ellen Meelkop ${ }^{1, \dagger}$, Liesbet Temmerman ${ }^{1}$, Nick \\ Suetens $^{1}$, Suzanne Rademakers ${ }^{2}$, Gert Jansen ${ }^{2}$, Liliane Schoofs ${ }^{1{ }^{*}}$
}

\begin{abstract}
Affiliations:
${ }^{1}$ Department of Biology, Functional Genomics and Proteomics Unit, KU Leuven, 3000 Leuven, Belgium.

${ }^{2}$ Department of Cell Biology, Erasmus MC, 3000 CA Rotterdam, the Netherlands.

${ }^{\dagger}$ Present address: Queensland Brain Institute, The University of Queensland, Brisbane, Australia.

*To whom correspondence should be addressed. E-mail: liliane.schoofs@bio.kuleuven.be
\end{abstract}




\begin{abstract}
:
Vasopressin- and oxytocin-related neuropeptides are key regulators of animal physiology, including water balance and reproduction. Although these neuropeptides also modulate social behavior and cognition in mammals, the mechanism for influencing of behavioral plasticity and the evolutionary origin of these effects are not well understood. Here, we present a functional vasopressin- and oxytocin-like signaling system in the nematode Caenorhabditis elegans. Through activation of its receptor NTR-1, a vasopressin/oxytocin-related neuropeptide, designated nematocin, facilitates the experience-driven modulation of salt chemotaxis, a type of gustatory associative learning in $C$. elegans. Our study suggests that vasopressin and oxytocin neuropeptides have ancient roles in modulating sensory processing in neural circuits that underlie behavioral plasticity.
\end{abstract}


The neurohypophyseal peptides vasopressin (VP) and oxytocin (OT) are related key hormones that regulate mammalian physiology (1-3). Their gene origin dates back at least 700 million years as indicated by the presence of structurally related peptides in some invertebrate phyla (4, 5). Peripheral effects of VP/OT-related peptides, primarily on water homeostasis and reproduction, are equally conserved $(5,6)$. Mammalian VP and OT peptides can act as central nervous system mediators of social behaviors, including parental care, pair bonding, social cognition and aggression $(7,8)$. They also modulate vertebrate cognition in a non-social context, although mechanistic complexities confound a clear understanding of these effects (9). Here, we identify and study a VP/OT-related system in the genetically tractable model Caenorhabditis elegans, which displays a high level of behavioral plasticity despite its relatively simple nervous system (10).

Through in silico data mining of the C. elegans genome, we characterized 91 presumptive neuropeptide heterotrimeric guanine nucleotide-binding protein ( $G$ protein)-coupled receptor (GPCR) genes (11). Protein sequences of two orphan rhodopsin class GPCR genes, which we named nematocin receptors $n t r-1$ (T07D10.2) and $n t r-2$ (F14F4.1), clustered in the VP and OT receptor clade (Fig. S1, Table S1). Sequence alignment with insect, mollusk, and mammalian VP/OT receptors revealed the presence of specific amino acid residues important for VP/OT peptide binding (Fig. S2, Table S1).

To determine the cognate ligand of NTR-1 and NTR-2, we cloned and transiently expressed each receptor in Chinese hamster ovary (CHO) cells stably overexpressing apo-aequorin and the promiscuous $\mathrm{G \alpha}_{16}$ subunit (12). We challenged these cells with a synthetic library of 262 known and predicted C. elegans peptides. NTR-1 expressing cells responded dose-dependently with a nanomolar half macimal effective concentration $\left(E_{50}\right)$ to a single peptide 
CFLNSCPYRRYamide, henceforward named nematocin (Fig. 1A). Several amino acid residues of nematocin match the neurohypophyseal peptide motif, supporting that it belongs to the VP/OT peptide family (Table S2). Structural conservation is also evident at the level of its preproprotein (Fig. 1B, S3, Table S3) encoded by the nematocin precursor gene ntc-1 (F39C12.4). Similar to the architecture of VP/OT-related precursors, NTC-1 comprises a cysteine-rich neurophysin domain located immediately downstream of the mature peptide. Insect and octopus VP/OTrelated peptides and a predicted, truncated form of nematocin, CFLNSCPY, were unable to activate NTR-1 (Fig. 1A, S4), indicating the importance of the C-terminal nematocin residues for receptor activation. NTR-2 did not respond to nematocin or affect the dose-dependent activation of NTR-1 (Fig. S5). We conclude that the VP/OT-related nematocin peptide is the likely cognate ligand of the C. elegans NTR-1 receptor.

We investigated intracellular signaling of NTR-1 by measuring the calcium and cAMP responses in NTR-1 expressing cells that lacked $G \alpha_{16}$ or coexpressed the CRE luciferase reporter. Both second messenger levels increased upon nematocin administration (Fig. S6), indicating that the NTR-1 receptor can signal through both calcium and cAMP messengers, similar to its mammalian VP and OT receptor counterparts (13).

To explore the cells and tissues involved in nematocin signaling, we observed full-length GFPtagged NTR-1 receptor and NTC-1 precursor proteins in transgenic C. elegans. We found that ntc-1 is expressed in thermosensory AFD neurons, neurosecretory NSM cells, AVK interneurons, the pharyngeal neuron M5 and the mechanosensory DVA neuron (Fig. 1C-D). The $n t r-1$ gene is strongly expressed in the left ASE (ASEL) gustatory neuron, the chemosensory neuron pairs ASH and ADF and most likely the PQR tail neuron (Fig. 1E-F, complete ntc-1, ntr- 
1 and $n t r-2$ expression patterns are reported in Fig. S7 and Movie S1-S3). These expression patterns suggest a role for nematocin signaling in modulating sensory neural circuits.

Since the ntr-1 expressing ASEL, ADF, and ASH neurons perform critical functions in chemotaxis of $C$. elegans towards water-soluble (gustatory) cues $(14,15)$, we studied the salt chemotaxis behavior of mutants defective in nematocin signaling. Null mutants for nematocin or its receptor showed wild type attraction to low $\mathrm{NaCl}$ concentrations and avoidance of high osmotic strength (Fig. S8). Hence, defects in the nematocin signaling pathway do not compromise the normal detection of $\mathrm{NaCl}$ and subsequent attractive or aversive responses.

Next, we investigated whether nematocin plays a more subtle role in sensory processing and focused on gustatory plasticity, which implies a change in salt chemotaxis behavior based upon prior experience $(16,17)$. We subjected mutants lacking nematocin or its receptor to this associative learning paradigm using a short-term gustatory plasticity assay. Chemotaxis towards the attractant $\mathrm{NaCl}$ was compared between naive worms and animals that were shortly (15 min) pre-exposed to the attractant in the absence of food (an aversive stimulus) (Fig. S9). Although pre-exposed wild type worms showed reduced attraction to or even avoidance of $\mathrm{NaCl}$, this aversive response was significantly reduced in $n t c-1$ and $n t r-1$ mutants (Fig. 2A). These results suggest that defects in the nematocin signaling cascade disrupt gustatory associative learning. To dissect part of the cellular circuit behind this effect, we generated transgenic lines expressing $n t r-$ 1 or $n t c-1$ under promoters specific to selected target cells. Cell-specific expression of $n t r-1$ in the gustatory ASEL neuron and of the nematocin precursor ntc-1 in AVK neurons rescued the plasticity defect of $n t r-1$ and $n t c-1$ mutants, respectively (Fig. 2B-C, S10). Our findings imply that nematocin, originating at least partly from the AVK interneurons, facilitates gustatory associative learning through NTR-1 mediated signaling in the ASEL sensory neuron, which has 
previously been found essential for salt attractive behaviors and gustatory plasticity (18). Null mutants for $n t r-2$ showed wild type gustatory plasticity, whereas the plasticity defects of $n t r-1$; ntr-2 double mutants resembled that of the $n t r-1$ single mutant (Fig. S11B).

Starvation prior to the learning assay is known to enhance gustatory plasticity of wild type worms, resulting in stronger aversive responses (17). Starved nematocin signaling mutants, however, showed wild type gustatory plasticity (Fig. S11), indicating that starvation triggers a nematocin independent mechanism to induce $\mathrm{NaCl}$ avoidance after pre-exposure.

Previous work revealed that $G$ protein as well as calcium signaling, via the Gy subunit GPC-1 and the TRPV channel OSM-9, amongst others, regulate gustatory plasticity (18). We generated double mutants for $n t r-1$ and $n t c-1$ with the gpc-1 or osm-9 genes. Their plasticity resembled that of single mutants (Fig. S12A-B), indicating that nematocin functions in the same genetic pathway. We then investigated whether nematocin interacts with dopamine and/or serotonin neurotransmitters, which play important roles in associative learning in mammals and C. elegans $(17,19,20)$. Nematocin signaling mutants also lacking serotonin or dopamine biosynthesis due to mutations in the tph-1 or cat-2 genes (17), respectively, showed no additive gustatory plasticity defects (Fig. S12C-D). Considering the severe plasticity defect of the cat-2 single mutant, the behaviors of double mutants carrying the cat-2 loss-of-function allele were deemed less conclusive (Fig. S12D). Based on our finding that nematocin acts in the same genetic pathway as tph-1, and probably also cat-2, we tested whether exogenous serotonin or dopamine could restore gustatory plasticity of $n t r-1$ mutants. Short (4 hours) or long (72 hours) term exposure to these neurotransmitters partially restored gustatory plasticity of mutants lacking the NTR-1 receptor (Fig. 2D-E), whereas naive responses of mutants and the behaviors of wild type 
animals were unaffected (Fig. S13). These results suggest that nematocin receptor signaling interacts with serotonergic and dopaminergic neurotransmission in gustatory plasticity.

The ability of animals to monitor environmental cues and adapt their behavior accordingly is crucial for their survival. We have shown that in C. elegans, VP/OT-related signaling is critical for gustatory associative learning, in line with the emergence of VP and OT as key regulators of mammalian cognition and behavior $(8,21)$. Our results indicate that VP and OT neuropeptides have ancient roles in modulating sensory processing in central neural circuits underlying behavioral plasticity. Hence, this neuropeptide signaling system likely arose when animals became mobile and started to make experience-based decisions, which happened prior to the divergence of protostomes and deuterostomes more than 700 million years ago. 


\section{References and Notes:}

1. M. S. Soloff, M. Alexandrova, M. J. Fernstrom, Oxytocin receptors: triggers for parturition and lactation? Science 204, 1313-1315 (1979).

2. W. Rosenthal et al., Molecular identification of the gene responsible for congenital nephrogenic diabetes insipidus. Nature 359, 233-235 (1992).

3. M. Schumacher, H. Coirini, D. W. Pfaff, B. S. McEwen, Behavioral effects of progesterone associated with rapid modulation of oxytocin receptors. Science 250, 691694 (1990).

4. E. Stafflinger et al., Cloning and identification of an oxytocin/vasopressin-like receptor and its ligand from insects. Proc. Natl. Acad. Sci. U. S. A 105, 3262-3267 (2008).

5. R. E. van Kesteren et al., A novel G protein-coupled receptor mediating both vasopressin- and oxytocin-like functions of Lys-conopressin in Lymnaea stagnalis. Neuron 15, 897-908 (1995).

6. M. J. Aikins et al., Vasopressin-like peptide and its receptor function in an indirect diuretic signaling pathway in the red flour beetle. Insect Biochem. Mol. Biol. 38, 740-748 (2008).

7. L. J. Young, Z. Wang, The neurobiology of pair bonding. Nat. Neurosci. 7, 1048-1054 (2004). 
8. A. Meyer-Lindenberg, G. Domes, P. Kirsch, M. Heinrichs, Oxytocin and vasopressin in the human brain: social neuropeptides for translational medicine. Nat. Rev. Neurosci. 12, 524-538 (2011).

9. D. de Wied, M. Diamant, M. Fodor, Central nervous system effects of the neurohypophyseal hormones and related peptides. Front. Neuroendocrinol. 14, 251-302 (1993).

10. E. L. Ardiel, C. H. Rankin, An elegant mind: learning and memory in Caenorhabditis elegans. Learn. Mem. 17, 191-201 (2010).

11. T. Janssen, M. Lindemans, E. Meelkop, L. Temmerman, L. Schoofs, Coevolution of neuropeptidergic signaling systems: from worm to man. Ann. N. Y. Acad. Sci. 1200, 1-14 (2010).

12. Materials and methods are available as supplementary materials on Science Online.

13. H. Orcel et al., Differential coupling of the vasopressin V1b receptor through compartmentalization within the plasma membrane. Mol. Pharmacol. 75, 637-647 (2009).

14. C. I. Bargmann, H. R. Horvitz, Chemosensory neurons with overlapping functions direct chemotaxis to multiple chemicals in C. elegans. Neuron 7, 729-742 (1991).

15. J. G. Culotti, R. L. Russell, Osmotic avoidance defective mutants of the nematode Caenorhabditis elegans. Genetics 90, 243-256 (1978). 
16. G. Jansen, D. Weinkove, R. H. Plasterk, The G-protein gamma subunit gpc-1 of the nematode C. elegans is involved in taste adaptation. EMBO J. 21, 986-994 (2002).

17. R. K. Hukema, S. Rademakers, G. Jansen, Gustatory plasticity in C. elegans involves integration of negative cues and $\mathrm{NaCl}$ taste mediated by serotonin, dopamine, and glutamate. Learn. Mem. 15, 829-836 (2008).

18. R. K. Hukema, S. Rademakers, M. P. Dekkers, J. Burghoorn, G. Jansen, Antagonistic sensory cues generate gustatory plasticity in Caenorhabditis elegans. EMBO J. 25, 312322 (2006).

19. P. Redgrave, K. Gurney, The short-latency dopamine signal: a role in discovering novel actions? Nat. Rev. Neurosci. 7, 967-975 (2006).

20. A. Izquierdo et al., Impaired reward learning and intact motivation after serotonin depletion in rats. Behav. Brain Res. 233, 494-499 (2012).

21. C. McCall, T. Singer, The animal and human neuroendocrinology of social cognition, motivation and behavior. Nat. Neurosci. 15, 681-688 (2012).

22. We thank the Caenorhabditis Genetics Center, S. Mitani, C. Bargmann and M. Alkema for strains and reagents; L. Vanden Bosch, M. Vanhove and W. De Haes for technical assistance. I.B., T.J. and L.T. benefit from the Fund for Scientific Research-Flanders (FWO) and E.M. from the KU Leuven Faculty Research Fund. This work was supported by the KU Leuven concerted research grant GOA/11/002 and FWO grants G.0767.09 and G.0601.11. GenBank accession numbers of ntc-1, ntr-1 and ntr-2 sequences are JQ277480, JQ277731 and JQ277479. Strains carrying the tm2385, tm2765 and tm2243 
alleles are available through the C. elegans National BioResource Project (NBRP), subject to a MTA. 

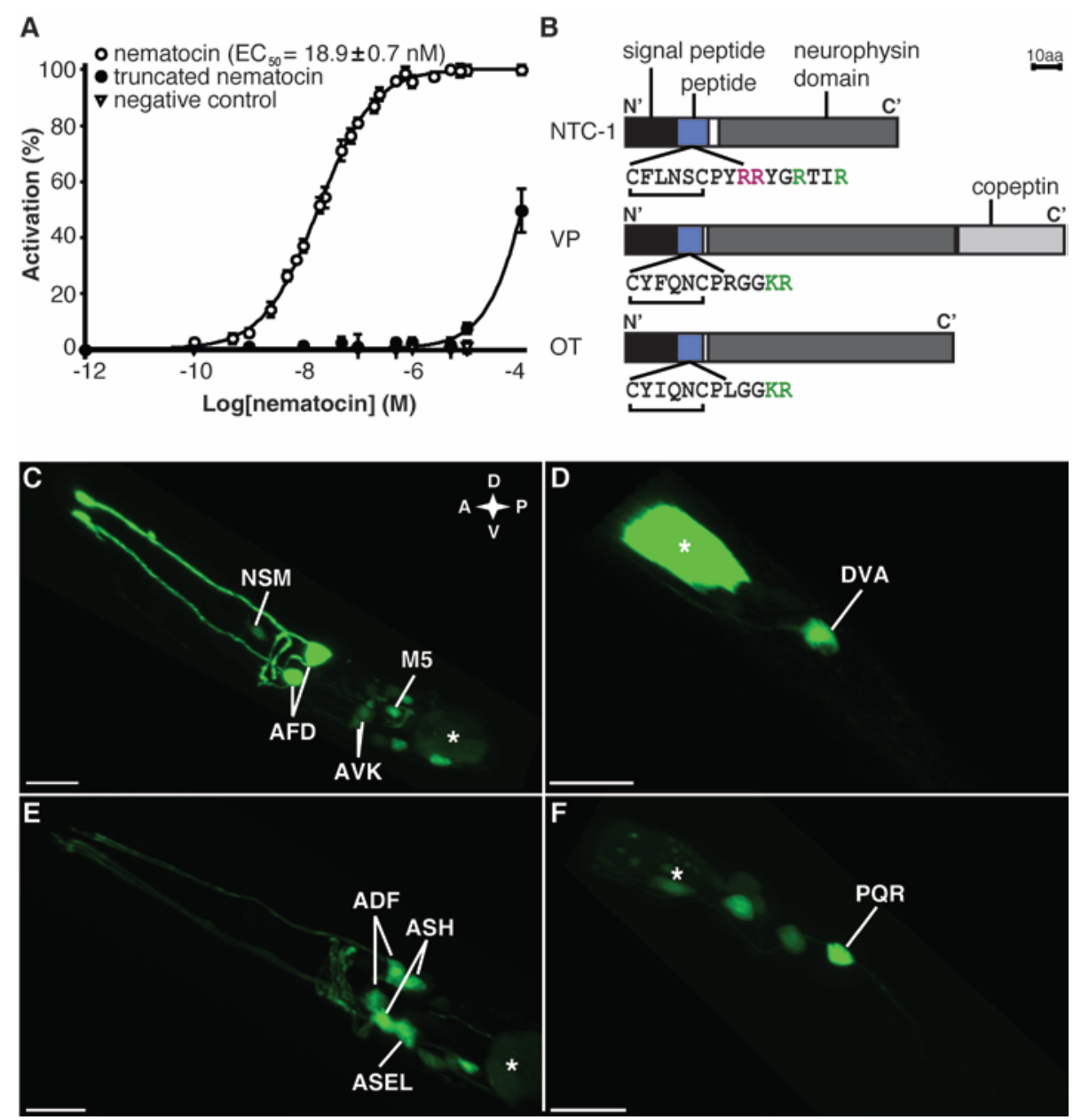

Fig. 1. ntc-1 encodes a VP/OT-related peptide, nematocin, that signals through the NTR-1 receptor. (A) Dose-response curves for calcium responses evoked by nematocin (CFLNSCPYRRYamide) and its C-terminally truncated variant CFLNSCPY in CHO cells either expressing NTR-1 or transfected with an empty vector (negative control). Each point ( \pm SEM) represents the average of two independent experiments performed in triplicate. Dose-response data are shown as relative (\%) to the highest value (100\% activation) after normalization to the maximum calcium response. (B) Domain structures of NTC-1, human VP and OT. Predicted proprotein convertase sites, purple and green; N’, amino-terminus; C’, carboxy-terminus. (C-D) Expression of $n t c-1:: g f p$ reporter transgene. (E-F) Expression of $n t r-1:: g f p$ transgene. (C-F) Left and right panels are labeled confocal Z-stack projections of the head and tail region (L1 wild 
type hermaphrodite), respectively. Asterisks mark fluorescence in the intestine, resulting from the co-injection marker Pelt-2::mCherry. Scale bars represent $10 \mu \mathrm{m}$.

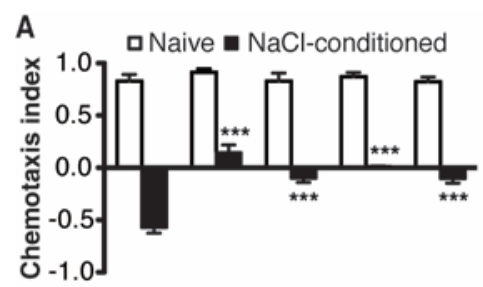

B

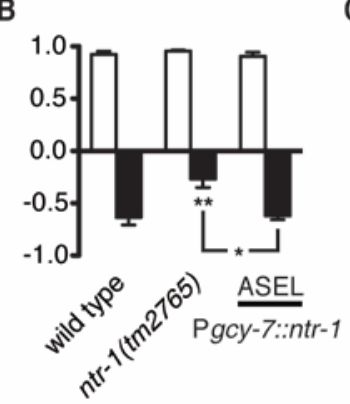

C

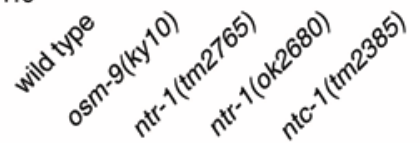

D

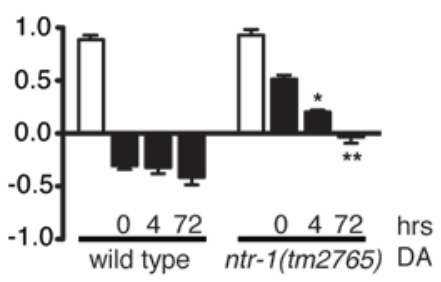

E

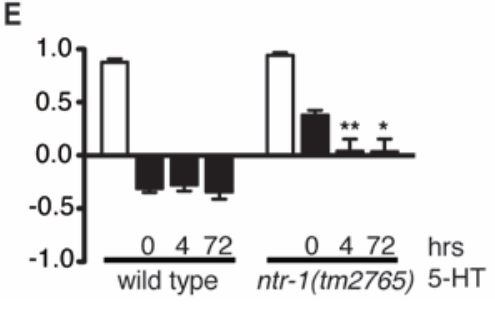

Fig. 2. Nematocin signaling regulates gustatory plasticity. (A) Gustatory plasticity of ntr-1 and ntc-1 mutants. The mean chemotaxis index towards $25 \mathrm{mM} \mathrm{NaCl}$ of animals pre-exposed in buffer with (conditioned) or without (naive) $\mathrm{NaCl}$ is plotted. osm-9 mutants were used as positive control. (B-C) $n t r-1$ or $n t c-1$ were expressed by cell-specific promoters in the $n t r-$ 1(tm2765) or ntc-1(tm2385) background, respectively, and gustatory plasticity of transgenic animals was tested. (D-E) Responses of wild type and ntr-1(tm2765) animals after 4 or $72 \mathrm{~h}$ of culture on plates containing $2 \mathrm{mM}$ dopamine (DA) or serotonin (5-HT). Open and filled bars represent naive and $\mathrm{NaCl}$-conditioned behaviors, respectively. For (A-C), responses were compared to wild type (unless indicated otherwise) by one-way ANOVA and Tukey post-hoc comparison. For (D-E), statistical significance scores refer to the relative change in mutant behavior compared to wild type changes upon dopamine/serotonin exposure and were based on 
coefficients of a fitted linear model. ${ }^{*} P<0.05$, ${ }^{* *} P<0.01$, $* * * P<0.001$; error bars indicate SEM ( $\mathrm{n} \geq 4$ assays). 


\section{Supplementary Materials:}

Materials and Methods

Figures S1-S13

Tables S1-S3

Movies S1-S3

References (22-65) 